\title{
SCEM Approach for singularly perturbed linear turning mid-point problems with an interior layer
}

\author{
M. Tarik Atay, Suleyman Cengizci and Aytekin Eryilmaz \\ Department of Mechanical Engineering, Abdullah Gul University, Kayseri, Turkey \\ Institute of Applied Mathematics, Middle East Technical University, Ankara, Turkey \\ Department of Mathematics, Nevsehir Haci Bektas Veli University, Nevsehir, Turkey
}

Received: 20 August 2015, Revised: 3 December 2015, Accepted: 22.January 2016

Published online: 30 January 2016.

\begin{abstract}
In this work, we study an important class of singular perturbation problems: singularly perturbed two-point turning point problems (TPP's) in ordinary differential equations (ODE's) exhibiting interior layer at the mid-point of their intervals. We consider this type of problems as two different singular perturbation problems which have a boundary layer at an end point of their intervals. This study is devoted to the Successive complementary expansion method (SCEM) to solve these two sub-problems. Two test problems are considered to check the efficiency and accuracy of the proposed method. Our numerical experiments show that SCEM approximations are in good agreement with exact and previously obtained solutions.
\end{abstract}

Keywords: Asymptotic approximation, interior layer, singular perturbation, successive complementary expansion method.

\section{Introduction}

Singular perturbation problems are differential equations in which a small positive parameter $0<\varepsilon \ll 1$ exists as a coefficient of the highest derivative term. The most significant property of this type of problems is the reduction of order of the equation as $\varepsilon \rightarrow 0$. Therefore, the solution involves a thin layer in which the solution exhibits rapid and interesting behaviours.

Singularly perturbed differential equations are often arise in mathematical modelling of physical and engineering problems, especially in fluid and celestial mechanics. They also arise in mechanical and electrical systems, solid mechanics, optimal control, reaction-diffusion processes, quantum physics, chemical/biochemical reactions and financial mathematics [2]. Some of the conventional asymptotic methods to approximate to the solution of these problems are the Method of Multiple Scales, the Method of Wentzel-Kramers-Brillouin Approximation [8,9], the Method of Matched Asymptotic Expansions (MMAE) [10], the Poincaré-Lindstedt Method and the Periodic Averaging Method [25]. Kadalbajoo and Gupta made a great survey and investigated the development of the conventional and novel methods to solve singular perturbation problems in [1]. In another great study [2], Parul gave some examples to these kinds of problems occuring in almost all science branches and briefly examined the conventional methods. Various numerical approaches also were applied to approximate to the solutions of turning point problems such as finite difference methods [5], finite element method [6,7], numerical integration method [11], initial value techniques [12] and Reproducing Kernel Method [13,14]. In the detailed work [15], Sharma et al. review the theory and methods, and study the development covering the years 1970-2011. 
In this paper, we study an important class of the singular perturbation problems: turning point problems (TPP's) in second order ordinary differential equations. General form of this kind of problems can be given as

$$
\begin{aligned}
\varepsilon y^{\prime \prime}+a(x) y^{\prime}-b(x) y & =g(x), x \varepsilon\left[x_{1}, x_{2}\right] \\
y\left(x_{1}\right) & =\alpha_{1}, y\left(x_{2}\right)=\alpha_{2}
\end{aligned}
$$

where $\varepsilon$ is a small parameter, $a(x)$ and $b(x)$ are sufficiently smooth, $g(x) \varepsilon C^{1}\left[x_{1}, x_{2}\right]$ and $\alpha_{1}, \alpha_{2} \varepsilon \mathbb{R}$. If $a(x)$ does not have a root in $\left[x_{1}, x_{2}\right]$, that is, if it does not change sing in the interval, then the solution of this problem has a boundary layer at either end-point of the interval as $\varepsilon \rightarrow 0$. If $a(x)$ has a root at $x=x_{0} \varepsilon\left[x_{1}, x_{2}\right]$, then the problem is called simple turning point problem, if it has multiple root in this interval it is called multiple turning point problem. All the distinct roots of $a(x)$ are called turning points of (1).

We deal with simple turning point problems in this study and consider the mid-point $x_{0}=\frac{x_{2}+x_{1}}{2} \varepsilon\left[x_{1}, x_{2}\right]$ as the turning point. We need some conditions that (1) has to satisfy as given in [3]

$$
\begin{aligned}
a\left(x_{0}\right) & =0\left(x_{0} \text { is turning point }\right) \text { and } \\
a^{\prime}\left(x_{0}\right) & >0\left(\text { no boundary layer, interior layer at } x_{0}\right),
\end{aligned}
$$

the maximum principle

$$
b(x)>0 \text { and } b\left(x_{0}\right)>0,
$$

to ensure the uniqueness of turning point

$$
\left|a^{\prime}(x)\right|>\left|\frac{a^{\prime}\left(x_{0}\right)}{2}\right|, x \varepsilon\left[x_{1}, x_{2}\right]
$$

and the condition to avoid resonance phenomena as given in [4]

$$
b(x)>\beta>0 ; \beta \varepsilon \mathbb{R}, x \varepsilon\left[x_{1}, x_{2}\right] .
$$

These conditions guarentee that the problem (1) has unique solution exhibiting an interior layer. If we assume that $b(x)=$ $g(x) \equiv 0$, as we later show,

$$
\begin{aligned}
\varepsilon y^{\prime \prime}+a(x) y^{\prime} & =0, x \varepsilon\left[x_{1}, x_{2}\right] \\
y\left(x_{1}\right) & =\alpha_{1}, y\left(x_{2}\right)=\alpha_{2}
\end{aligned}
$$

has a fixed turning point $\left(x_{0}, y_{0}\right)$ for each different $\varepsilon$ value. This special case helps us to divide the problem into two sub-problems.

So far, various numerical and asymptotic methods was employed to approximate to the solutions of turning point problems. Some of them can be given as : finite difference methods[5], finite element methods[6,7], the Method of 
(WKB) Approximation [8,9], the Method of Matched Asymptotic Expansions (MMAE)[10], numerical integration methods[11], initial value techniques[12] and reproducing kernel methods (RKM)[13,14]. In the detailed work [15], Sharma et al. review the theory and methods, and study the development covering the years 1970-2011.

We consider the problem (1.1) as two boundary layer problems and using Successive Complementary Expansion Method (SCEM) obtain two uniformly valid approximation over each sub-domain. It means that, considering the mid-point $x=x_{0} \varepsilon\left[x_{1}, x_{2}\right]$ as the turning point, we reach two sub-problem and corresponding uniformly valid approximations over the intervals $\left[x_{1}, x_{0}\right]$ and $\left[x_{0}, x_{2}\right]$.

The remainder sections of our study continue as follows: In Section 2, we describe the present method (SCEM) that is developed by J. Mauss and J. Cousteix in $[16,17]$ and to implement it to the turning point problems we modify it. In section 3, we employe an illustrative example to explain how the method works. In section 4, we solve two interesting turning point problems (TPP's) existing in the literature and in the conclusions, we compare the results with previously obtained by other methods.

\section{Description of the method}

In this section, we give some basic concepts about the asymptotic expansions and SCEM.

Consider the functions $\phi(x, \varepsilon)$ and $\phi_{a}(x, \varepsilon)$ defined in a domain $D$ are asymptotically identical to order $\delta(\varepsilon)$ if the difference is asymptotically smaller than $\delta(\varepsilon)$, i.e., $\phi(x, \varepsilon)-\phi_{a}(x, \varepsilon)=o(\delta(\varepsilon))$, where $\delta(\varepsilon)$ is an order function and $\varepsilon$ is control parameter arising from the physical problem under consideration. The function $\phi_{a}(x, \varepsilon)$ is called an asymptotic approximation of the function $\phi(x, \varepsilon)$. Asymptotic approximations, in general, are defined as

$$
\phi_{a}(x, \varepsilon)=\sum_{i=0}^{n} \delta_{i}(\varepsilon) \varphi_{i}(x, \varepsilon)
$$

where the asymptotic sequence of order functions $\delta_{i}(\varepsilon)$ an asymptotic sequence such that $\delta_{i+1}(\varepsilon)=o\left(\delta_{i}(\varepsilon)\right)$, as $\varepsilon \rightarrow 0$ and all the functions $\varphi_{i}(x, \varepsilon)$ are of strict order $1, \varphi_{i}=O_{S}(1)$. Under these conditions the approximation (5) is called generalized asymptotic expansion. If the expansion is written down in the form of

$$
\phi_{a}(x, \varepsilon)=E_{0} \phi=\sum_{i=0}^{n} \delta_{i}^{(0)}(\varepsilon) \varphi_{i}^{(0)}(x),
$$

then it is named as regular asymptotic expansion. The special operator $E_{0}$ called the outer expansion operator at a given order $\delta(\varepsilon)$, thus we obtain $\phi-E_{0} \phi=o(\delta(\varepsilon))$.

Interesting behaviours occur when the function is not regular in $D$, so the region $D_{0} \in D$ in which (6) is valid is called outer region. This is the boundary (or interior) layer effect and we must introduce boundary layer domains. We introduce an inner domain which can be formally denoted $D_{1}=D-D_{0}$ and located near the origin (here we assume that the boundary layer occurs near the origin $x=0)$. The boundary layer variable is $\bar{x}=\frac{x}{\xi(\varepsilon)}, \xi(\varepsilon)$ being the order of thickness of this boundary layer. If a regular expansion can be constructed in $D_{1}$, we can write

$$
\phi_{a}(x, \varepsilon)=E_{1} \phi=\sum_{i=0}^{n} \delta_{i}^{(1)}(\varepsilon) \varphi_{i}^{(1)}(\bar{x}) .
$$


The inner expansion operator $E_{1}$ is defined in $D_{1}$ at the same order $\delta(\varepsilon)$ as the outer expansion operator $E_{0}$; thus, $\phi-E_{1} \phi=o(\delta(\varepsilon))$ and so

$$
\phi_{a}=E_{0} \phi+E_{1} \phi-E_{1} E_{0} \phi
$$

is clearly uniformly valid approximation to order $\delta(\varepsilon)$ [16]-[19] and as it can be seen in detail in [18] satisfying $E_{1} E_{0} \phi=$ $E_{0} E_{1} \phi$. This is the main idea underlying the MMAE. It is based on finding two different approximations for different two regions, which are called inner region (where the solution exhibits rapid changes) and outer region (which is far from the inner region). At the last stage of the method, these two approximations are matched using the limit process to obtain a uniformly valid approximation. More detailed and advanced information can be found in [20]-[26]. Sometimes the matching procedure may be tiresome or even impossible. Therefore, we present and examine an efficient method socalled Successive Complementary Expansion Method (SCEM). In SCEM, instead of finding two different approximations to match, at the first stage, a uniformly valid approximation that satisfies the boundary conditions exactly is assumed. So, using the SCEM we will not be in need of matching and directly reach exactly satisfied boundary conditions. The uniformly valid SCEM approximation is in the regular form

$$
y_{n}^{\text {scem }}(x, \bar{x}, \varepsilon)=\sum_{i=0}^{n} \delta_{i}(\varepsilon)\left[y_{i}(x)+\Psi_{i}(\bar{x})\right]
$$

where $\delta_{i}(\varepsilon)$ is an asymptotic sequence and $\Psi_{i}(\bar{x})$ are the complementary approximations that depends on $\bar{x}$. Functions $y_{i}(x)$ are the outer approximation functions that have been found by MMAE and they only depend on $x$, not also on $\varepsilon$. In its regular form, SCEM is equivalent to MMAE. If $y_{i}(x)$ and $\Psi_{i}(\bar{x})$ also depend on $\varepsilon$, the uniformly valid SCEM approximation is named as generalized SCEM approximation and given as in the following form

$$
y_{n g}^{\text {scem }}(x, \bar{x}, \varepsilon)=\sum_{i=0}^{n} \bar{\delta}_{i}(\varepsilon)\left[\bar{y}_{i}(x, \varepsilon)+\bar{\Psi}_{i}(\bar{x}, \varepsilon)\right] .
$$

with

$$
y_{n g}^{\text {scem }}(x, \bar{x}, \varepsilon)=y_{n}^{s c e m}(x, \bar{x}, \varepsilon)+o\left(\delta_{n+1}\right)
$$

The sequence of order functions $\delta_{i}(\varepsilon)$ may or not be same with $\bar{\delta}_{i}(\varepsilon)$. In [17], a more detailed convergence analysis of the method has been done. Now we give implementation of SCEM to TTP's on a illustrative example. Dorr and Parter [27] show that the turning point problems which are in the form of

$$
\varepsilon y^{\prime \prime}(x)+a(x) y^{\prime}(x)=0, y\left(x_{1}\right)=\alpha_{1}, y\left(x_{2}\right)=\alpha_{2}, a \leq x \leq b
$$

have the solution in the form of

$$
y(x, \varepsilon)=\alpha_{1}+\left(\alpha_{2}-\alpha_{1}\right) \frac{\int_{x_{1}}^{x} G(\tau, \varepsilon) d \tau}{\int_{x_{1}}^{x_{2}} G(\tau, \varepsilon) d \tau},
$$

where

$$
G(\tau, \varepsilon)=\exp \left[\frac{-1}{\varepsilon} \int_{x_{1}}^{x} \tau a(\tau) d \tau\right]
$$

(c) 2016 BISKA Bilisim Technology 
If we consider the turning point as $x_{0}=\frac{x_{1}+x_{2}}{2}$ (mid-point), we reach

$$
y\left(x_{0}\right)=\alpha_{1}+\left(\alpha_{2}-\alpha_{1}\right) \frac{\int_{x_{1}}^{x_{0}} \exp \left(\frac{-1}{\varepsilon} \int_{x_{1}}^{x_{0}} \tau a(\tau) d \tau\right) d \tau}{\int_{x_{1}}^{x_{2}} \exp \left(\frac{-1}{\varepsilon} \int_{x_{1}}^{x_{0}} \tau a(\tau) d \tau\right) d \tau}=\frac{\alpha_{1}+\alpha_{2}}{2} .
$$

It means that, the turning point $\left(x_{0}, y_{0}\right) \in \mathbb{R}^{2}$ is a fixed point, so its placement does not depend on $\varepsilon$. In this manner, we consider the problem (1) as two boundary layer problems. These sub-problems can be given as

$$
\varepsilon y_{1}^{\prime \prime}(x)+p(x) y_{1}^{\prime}(x)=0, y_{1}(a)=\alpha_{1}, y_{1}\left(x_{0}\right)=\frac{\alpha_{1}+\alpha_{2}}{2},
$$

and

$$
\varepsilon y_{2}^{\prime \prime}(x)+p(x) y_{2}^{\prime}(x)=0, y_{2}(a)=\frac{\alpha_{1}+\alpha_{2}}{2}, y_{2}(x)=\alpha_{2} .
$$

As an illustrative example, consider the singularly perturbed problem that is given in [11]

$$
\varepsilon y^{\prime \prime}(x)+2 x y^{\prime}(x)=0, y(-1)=-1, y(1)=1 .
$$

This problem has an interior layer at $x=0$ and the exact solution is given by

$$
y(x)=\operatorname{erf}(x / \sqrt{\varepsilon}) .
$$

Let us consider the problem as two different boundary layer problems. Thus, we have two sub-problems:

$$
\begin{gathered}
\varepsilon y_{1}^{\prime \prime}(x)+2 x y_{1}^{\prime}(x)=0, y_{1}(-1)=-1, y_{1}(0)=0, \\
\varepsilon y_{2}^{\prime \prime}(x)+2 x y_{2}^{\prime}(x)=0, y_{2}(0)=0, y_{2}(1)=1 .
\end{gathered}
$$

Here, the problem (18) has right-end boundary layer and (19) has left-end boundary layer. Now, we use the SCEM to obtain uniformly valid approximations for each interval $[-1,0]$ and $[0,1]$, respectively for the problems (18) and (19). For the right-end boundary layer problem (the same procedure is valid for left-end boundary layer problem) one-term SCEM approximation is sought as follows:

$$
y_{\text {scemr }}(x, \bar{x}, \varepsilon)=y_{\text {outerr }}(x, \varepsilon)+\Psi_{r}(\bar{x}, \varepsilon) .
$$

For $\varepsilon=0$, the outer solution is found as a constant function $y_{\text {outerr }}(x)=-1$, so the SCEM approximation is

$$
y_{\text {scemr }}(x, \bar{x}, \varepsilon)=\Psi_{r}(\bar{x}, \varepsilon)-1 .
$$

If the equation (21) is substituted into the problem (16), the complementary approximation $\Psi_{r}(\bar{x}, \varepsilon)$ is found as

$$
\Psi_{r}^{\prime \prime}(\bar{x}, \varepsilon)+2 \varepsilon \bar{x} \Psi_{r}^{\prime}(\bar{x}, \varepsilon)=0
$$




\begin{tabular}{|c|c|c|c|}
\hline$x$ & Exact & SCEM & Abs. Error \\
\hline-0.20 & -1.000000000000 & -1.000000000000 & 0.000000000000 \\
\hline-0.15 & -0.999999999980 & -0.999999999980 & $1.22124532 e-15$ \\
\hline-0.10 & -0.999992255783 & -0.999992255751 & $3.25113269 e-11$ \\
\hline-0.05 & -0.974652681322 & -0.974652682387 & $1.06509390 e-09$ \\
\hline-0.03 & -0.820287505121 & -0.820287505683 & $5.62873192 e-10$ \\
\hline-0.01 & -0.345279153981 & -0.345279151308 & $2.67243371 e-09$ \\
\hline 0.00 & 0.000000000000 & 0.000000000000 & 0.000000000000 \\
\hline 0.01 & 0.345279153981 & 0.345279151308 & $2.67243371 e-09$ \\
\hline 0.03 & 0.820287505121 & 0.820287505683 & $5.62873192 e-10$ \\
\hline 0.05 & 0.974652681322 & 0.974652682387 & $1.06509390 e-09$ \\
\hline 0.10 & 0.999992255783 & 0.999992255751 & $3.25113269 e-11$ \\
\hline 0.15 & 0.999999999980 & 0.999999999980 & $1.22124532 e-15$ \\
\hline 0.20 & 1.000000000000 & 1.000000000000 & 0.000000000000 \\
\hline
\end{tabular}

Table 1: Numerical results for illustrative example, $\varepsilon=0.001$.

with the boundary conditions

$$
\begin{aligned}
& x=-1 \Rightarrow \bar{x}=\frac{x}{\varepsilon}=\frac{-1}{\varepsilon} \Rightarrow \Psi_{r}\left(-\frac{1}{\varepsilon}, \varepsilon\right)=0, \\
& x=0 \Rightarrow \bar{x}=\frac{x}{\varepsilon}=\frac{0}{\varepsilon}=0 \Rightarrow \Psi_{r}(0, \varepsilon)=1 .
\end{aligned}
$$

When the same procedure is applied for the left-end boundary layer problem, following results are obtained.

We concentrate on the points near the interior layer where the solution exhibits rapid changes. Table 1 confirms that even near the interior layer the present method works well.

\section{Numerical experiments}

In this section, two singularly perturbed turning point problems are given and the SCEM is applied to approximate to their solutions. We prefer problems that has exact solutions in order to compare the numerical results. Results are also supplied with figures and absolute errors. All the computations are performed by using Matlab2014a.

Example 1. Consider the singularly perturbed turning point problem given in [3]

$$
\varepsilon y^{\prime \prime}+x y^{\prime}=0,-1 \leq x \leq 1
$$

with the boundary conditions

$$
y(-1)=0, y(1)=2,
$$

and the exact solution to this problem is given by

$$
y(x)=1+\frac{\operatorname{erf}(x / \sqrt{2 \varepsilon})}{\operatorname{erf}(1 / \sqrt{2 \varepsilon})} .
$$

It is clear that the problem has turning point at the point $x=0$. 


\begin{tabular}{|c|c|c|c|}
\hline$x$ & Exact & SCEM & Abs. Error \\
\hline-0.20 & 0.045500263896 & 0.045500263890 & $6.07063704 e-12$ \\
\hline-0.15 & 0.133614402537 & 0.133614402536 & $1.22601928 e-12$ \\
\hline-0.10 & 0.317310507862 & 0.317310507869 & $6.65428823 e-12$ \\
\hline-0.05 & 0.617075077451 & 0.617075077451 & $7.82018894 e-12$ \\
\hline-0.03 & 0.764177155622 & 0.764177155627 & $5.42232925 e-12$ \\
\hline-0.01 & 0.920344325445 & 0.920344325447 & $1.93445259 e-12$ \\
\hline 0.00 & 1.000000000000 & 1.000000000000 & 0.000000000000 \\
\hline 0.01 & 1.079655674554 & 1.079655674552 & $1.93445259 e-12$ \\
\hline 0.03 & 1.235822844377 & 1.235822844372 & $5.42232925 e-12$ \\
\hline 0.05 & 1.382924922548 & 1.382924922540 & $7.82018894 e-12$ \\
\hline 0.10 & 1.682689492137 & 1.682689492130 & $6.65428823 e-12$ \\
\hline 0.15 & 1.866385597462 & 1.866385597463 & $1.22601928 e-12$ \\
\hline 0.20 & 1.954499736103 & 1.954499736109 & $6.07063704 e-12$ \\
\hline
\end{tabular}

Table 2: Numerical results for Example 4.1, $\varepsilon=0.01$.

\begin{tabular}{|c|c|c|c|}
\hline$x$ & Exact & SCEM & Abs. Error \\
\hline-0.20 & $2.53962850 e-10$ & $2.53965369 e-10$ & $2.51887455 e-15$ \\
\hline-0.15 & $2.10143595 e-06$ & $2.10143902 e-06$ & $3.06494037 e-12$ \\
\hline-0.10 & 0.001565402258 & 0.001565402318 & $6.03590465 e-11$ \\
\hline-0.05 & 0.113846298006 & 0.113846297644 & $3.61998361 e-10$ \\
\hline-0.03 & 0.342781711147 & 0.342781711593 & $4.46029213 e-10$ \\
\hline-0.01 & 0.751829634045 & 0.751829634588 & $5.42514255 e-10$ \\
\hline 0.00 & 1.000000000000 & 1.000000000000 & 0.000000000000 \\
\hline 0.01 & 1.248170365954 & 1.248170365411 & $5.42514255 e-10$ \\
\hline 0.03 & 1.657218288852 & 1.657218288406 & $4.46029213 e-10$ \\
\hline 0.05 & 1.886153701993 & 1.886153702355 & $3.61998361 e-10$ \\
\hline 0.10 & 1.998434597741 & 1.998434597681 & $6.03590465 e-11$ \\
\hline 0.15 & 1.999997898564 & 1.999997898560 & $3.06494037 e-12$ \\
\hline 0.20 & 1.999999999746 & 1.999999999746 & $2.51887455 e-15$ \\
\hline
\end{tabular}

Table 3: Numerical results for Example 4.1, $\varepsilon=0.001$.

In Table 2-3, we concentrate on the points near the interior layer $x_{0}=0$ where the solution exhibits rapid changes. [3,14] can be examined for further comparisons.

Example 2. Consider the singularly perturbed turning point problem given in [28]

$$
\varepsilon y^{\prime \prime}+\left(x-\frac{1}{2}\right) y^{\prime}=0,0 \leq x \leq 1
$$

with the boundary conditions

$$
y(0)=1, y(1)=3,
$$

and the exact solution to this problem is given by

$$
y(x)=2+\frac{\operatorname{erf}\left(\frac{2 x-1}{2 \sqrt{2 \varepsilon}}\right)}{\operatorname{erf}\left(\frac{1}{2 \sqrt{2 \varepsilon}}\right)} .
$$

It is clear that the problem has turning point at the point $x=\frac{1}{2}$. 


\begin{tabular}{|c|c|c|c|}
\hline$x$ & Exact & SCEM & Abs. Error \\
\hline 0.000 & 1.000000000000 & 1.000000000000 & 0.000000000000 \\
\hline 0.400 & 1.001565402258 & 1.001565402256 & $1.69686487 e-12$ \\
\hline 0.450 & 1.113846298006 & 1.113846297986 & $1.97870608 e-11$ \\
\hline 0.495 & 1.874367061162 & 1.874367061181 & $1.87414528 e-11$ \\
\hline 0.499 & 1.974772879369 & 1.974772879373 & $3.85069753 e-12$ \\
\hline 0.500 & 2.000000000000 & 2.000000000000 & 0.000000000000 \\
\hline 0.501 & 2.025227120630 & 2.025227120630 & $3.85069753 e-12$ \\
\hline 0.505 & 2.125632938837 & 2.125632938818 & $1.87414528 e-11$ \\
\hline 0.550 & 2.886153701993 & 2.886153702013 & $1.97870608 e-11$ \\
\hline 0.600 & 2.998434597741 & 2.998434597743 & $1.69908531 e-12$ \\
\hline
\end{tabular}

Table 4: Numerical results for Example 4.2, $\varepsilon=0.001$.

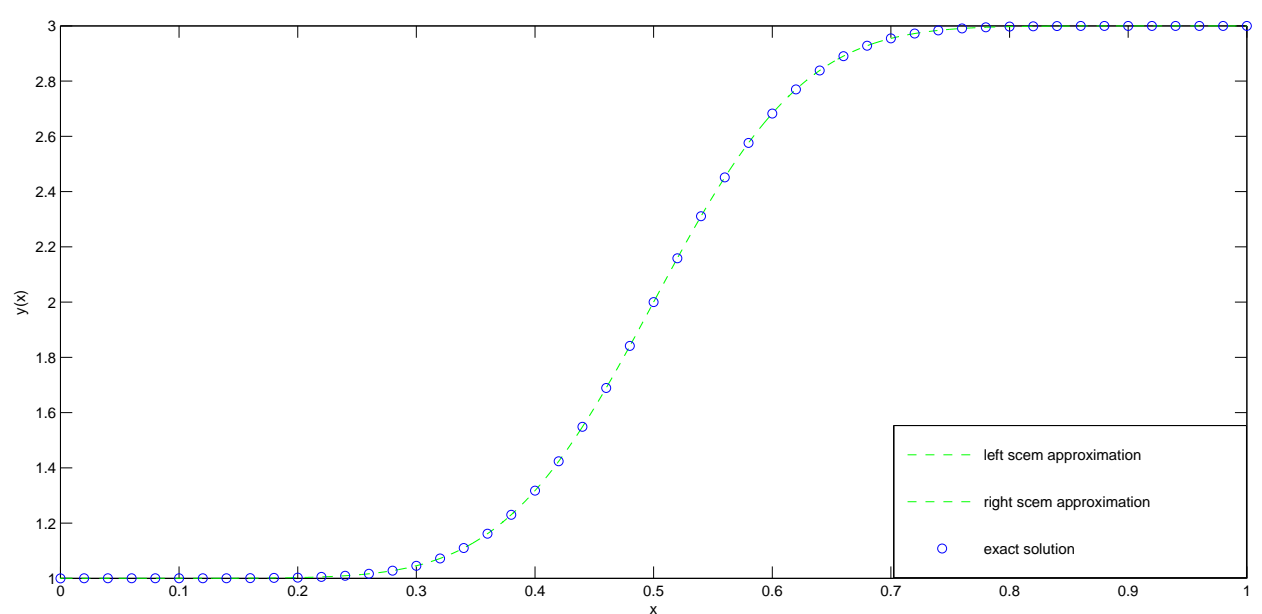

Fig. 1: Comparison of results for Example 4.2, $\varepsilon=0.01$.

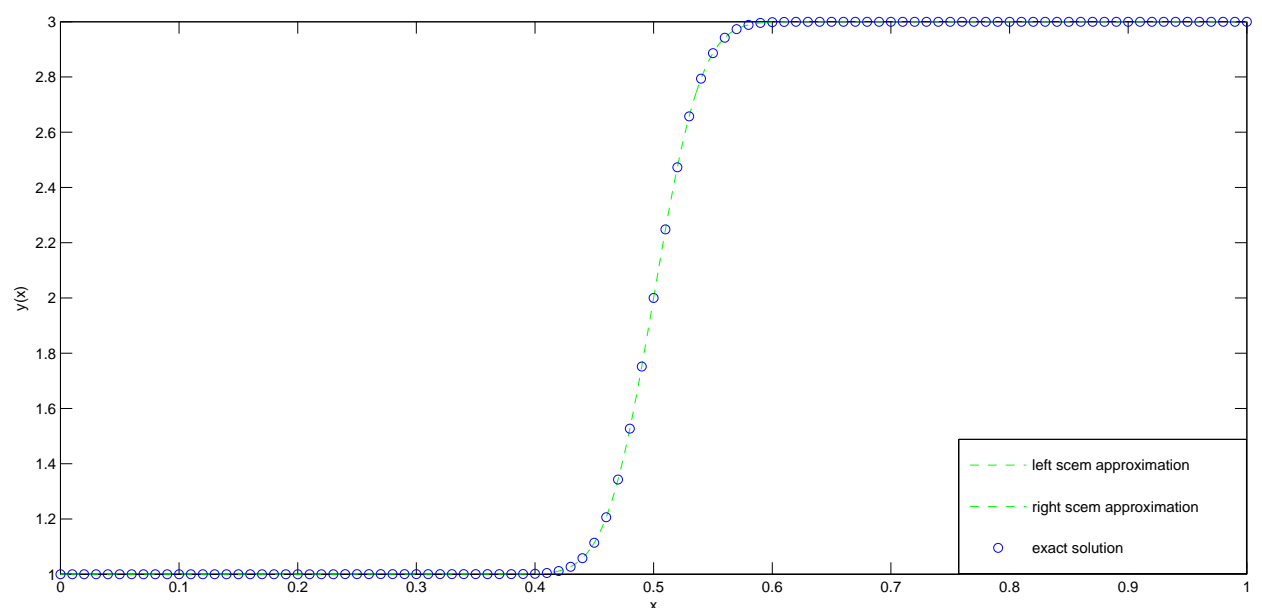

Fig. 2: Comparison of results for Example 4.2, $\varepsilon=0.001$.

In Table 4 and Fig. 1-3, we concentrate on the points near the interior layer $x_{0}=0.5$ where the solution exhibits rapid changes. 


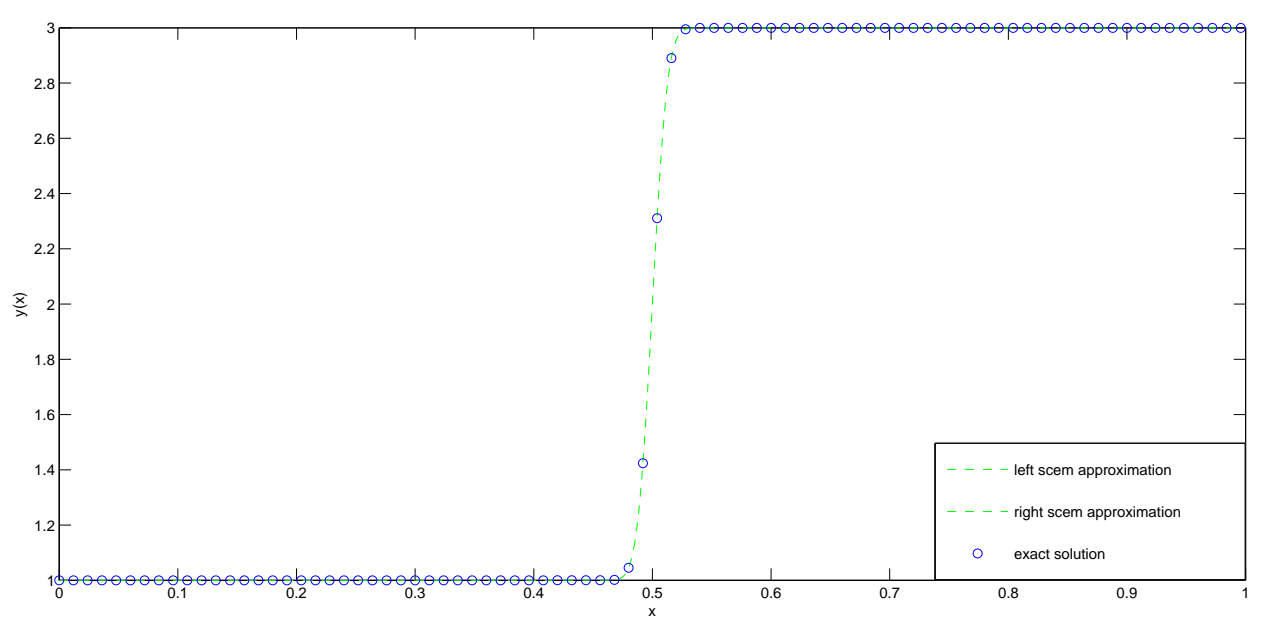

Fig. 3: Comparison of results for Example 4.2, $\varepsilon=0.0001$.

\section{Conclusions}

In this work, we investigate a class of linear turning point problems TPP's in ordinary differential equations which has an interior layer at the mid-point of the interval. We divide the problem into two sub-problems and apply SCEM which has been used to approximate to the solutions of boundary layer problems. In application of the method any matching procedure is required and the boundary conditions are satisfied exactly, but not asymptotically. Our numerical experiments that is supported with tables and figures show that SCEM is well-suited for this class of turning point problems.

\section{Competing interests}

The authors declare that they have no competing interests.

\section{Authors' contribution}

All authors contributed extensively in the development of this article. All authors read and approved the final manuscript.

\section{Acknowledgments}

The authors declare that they did not use any external fund, contribution or support.

\section{References}

[1] M.K. Kadalbajoo, V. Gupta, A brief survey on numerical methods for solving singularly perturbed problems, Appl. Math. Comput. 217 (2010) 3641-3716.

[2] K. Manoj, Methods for solving singular perturbation problems arising in science and engineering, Mathematical and Computer Modelling, 54 (1) (2011) 556-575.

[3] M.K. Kadalbajoo, K.C. Patidar, Variable mesh spline approximation method for solving singularly perturbed turning point problems having boundary layer(s), Comput. Math. Appl., 18.2 (2010) 207-220. 
[4] L.R. Abrahamsson, A priori estimates for solutions of singular perturbations with a turning point, Stud. Appl. Math. 56 (1977) $51-69$.

[5] C.G. Lange, R.M. Miura, Singular perturbation analysis of boundary-value problems for differential difference equations III. Turning point problems, SIAM Journal on Applied Mathematics 45 (1985) 708-734.

[6] E. O'Riordan, M. Stynes, A uniform finite element method for a conservative singularly perturbed problem, J. Comput. Appl. Math. 18 (2) (1987) 163-174.

[7] E. O'Riordan, M. Stynes, A uniformly accurate finite element method for a singularly perturbed one-dimensional reactiondiffusion problem, Math. Comput. 47 (176) (1986) 555- 570.

[8] M. Nakano, Second-order linear ordinary differential equations with turning points and singularities. I., Kodai Math. Sem. Rep. 29 (1-2) (1977) 88-102.

[9] M. Nakano, Second-order linear ordinary differential equations with turning points and singularities. II, Kodai Math. J. 1 (2) (1978) 304-312.

[10] A.M. Wazwaz, F.B. Hanson, Matched uniform approximations for a singular boundary and interior turning point, SIAM J. Appl. Math. 46 (6) (1986) 943-961

[11] K. Phaneendra, Y.N. Reddy, GBSL. Soujanya, Non-iterative numerical integration method for singular perturbation problems exhibiting internal and twin boundary layers, Int. J. Appl. Math. Comput. 3 (2011) 9-20.

[12] S.Natesan, N.Ramanujam, Initial value technique for singularly perturbed turning point problems exhibiting twin boundary layers, J.Optim.Theoret.Appl. 99 (1998) 37-52..

[13] F. Geng, S.P. Qian, Reproducing kernel method for singularly perturbed turning point problems having twin boundary layers, Appl. Math. Lett. 26 (2013) 998-1004.

[14] F. Geng, S.P. Qian, S. Li, A numerical method for singularly perturbed turning point problems with an interior layer, J. Comput. Appl. Math. 255 (2014) 97-105.

[15] K. K. Sharma, P. Rai, K. C. Patidar, A review on singularly perturbed differential equations with turning points and interior layers, Appl. Math. Comput. 219.22 (2013) 10575-10609.

[16] J. Mauss, J. Cousteix, Uniformly valid approximation for singular perturbation problems and matching principle, C. R. MVecanique 330 (10) (2002) 697-702.

[17] J. Cousteix, J. Mauss, Asymptotic Analysis and Boundary Layers, Scientific Computation, vol. XVIII, Springer, Berlin, Heidelberg, 2007.

[18] W. Eckhaus, Matched Asymptotic Expansions and Singular Perturbation,. North Holland Publishing Company, Amsterdam, The Netherlands, 1973.

[19] W. Eckhaus, Asymptotic analysis of singular perturbations, Studies in Mathematics and its Applications 9, North-Holland Publishing Co., Amsterdam-New York, 1979.

[20] A. H. Nayfeh, Perturbation methods, Pure and applied mathematics, JohnWiley \& sons, 1973.

[21] P. Lagerstrom, Matched Asymptotic Expansions: Ideas and Techniques, vol. 76 of Applied Mathematical Sciences, Springer, New York, NY, USA, 1988.

[22] E. J. Hinch, Perturbation Methods, Cambridge University Press, Cambridge, Mass, USA, 1991.

[23] M. Van Dyke, Perturbation Methods in Fluid Mechanics, Academic Press, New York, 1964.

[24] R. S. Johnson, Singular perturbation theory: Mathematical and analytical techniques with applications to engineering Springer Science \& Business Media, 2006.

[25] F. Verhulst, Methods and Applications of Singular Perturbations, Texts in Appl. Math., vol. 50, Springer, New York, 2005.

[26] M. H. Holmes, Introduction to Perturbation Methods, Springer, Berlin, 1995.

[27] F. W. Dorr, S. V. Parter, Singular perturbations of nonlinear boundary value problems with turning points, J. Math. Anal. Appl. 29 (1970) 273-293

[28] D. Kamowitz, Multigrid applied to singular pertubation problems, ICASE Report, 87-1, 1987. 